\title{
Consecuencias del cambio en el sexo legal en el sistema de salud colombiano*
}

\author{
Recibido: 2 de mayo de 2019 - Aprobado: 12 de agosto de 2019 \\ https://doi.org/10.22395/ojum.v19n38a3 \\ Adiley Carmona Montoya** \\ Christian David Rivera Aguirre***
}

\section{RESUMEN}

El presente artículo analiza las consecuencias que trae para el sistema de salud colombiano el cambio de la variable sexo en el documento de identidad de una persona transgénero, quien puede identificarse legalmente con un sexo, pero conservar la biología de otro. A partir de una investigación de tipo documental y exploratoria, fue posible concluir que para el sistema es importante la identidad biológica de las personas, ya que se utiliza para realizar gestión de riesgo en salud, y se usa para calcular los ingresos en capitación para las entidades promotoras de salud, por lo cual se recomienda tener esta variable en cuenta en el sistema.

Palabras clave: personas transgénero; capitación; gestión del riesgo; sistema de salud.

* Artículo resultado de la investigación titulada "Transgenerismo en el sistema de salud: Consecuencias económicas y en el modelo de atención de Colombia", inscrito a la línea de investigación Derecho Laboral, Seguridad Social y Responsabilidad Médica del Grupo de Estudios Jurídicos de Facultad de Derecho de la Universidad CES.

** Abogada, especialista en Gerencia de la Seguridad Social, magíster en Seguridad Social. Docente e investigadora, Grupo de Estudios Jurídicos, Universidad CES, Medellín, Colombia. Correo electrónico: acarmona@ces.edu.co Orcid: https://orcid.org/0000-0002-6607-7954

${ }^{* * *}$ Abogado, especialista en Gerencia de la Seguridad Social, magíster en Seguridad Social. Docente e investigador, Grupo de Estudios Jurídicos, Universidad CES. Correo electrónico: christianda123@ hotmail.com Orcid: https://orcid.org/0000-0002-1926-4255 


\section{Consequences of the Legal Sex Change in the Colombian Health System}

\section{ABSTRACT}

This article analyzes the consequences for the Colombian health system of the change of the sex variable in the ID of a transgender citizen who might legally identify with a sex while conserving its biology with another. From documental and exploratory research it was possible to conclude that the biological identity of people is what is relevant for the system given that it uses this information for health's risks management and also for calculating the capitation income for the health promotion entities (EPS in Spanish), whereby this variable is highly suggested for the system.

Keywords: transgender people; capitation; risk management; health system.

\section{Consequências da mudança de sexo legal no sistema de saúde colombiano}

\section{RESUMO}

Este artigo analisa as consequências que traz para o sistema de saúde colombiano a mudança de sexo no documento de identidade de uma pessoa transgênero, a qual é identificada legalmente com um sexo, mas conserva a biologia de outro. A partir de uma pesquisa de tipo documental e exploratória, foi possível concluir que, para o sistema, é importante a identidade biológica das pessoas, já que esta é utilizada para realizar o gerenciamento de risco em saúde e para calcular os ingressos em captação para as entidades promotoras de saúde . Por isso, recomenda-se considerar essa variável no sistema.

Palavras-chave: pessoas transgênero; capitação; gestão do risco; sistema de saúde. 


\section{INTRODUCCIÓN}

En Colombia no solo es posible realizar intervenciones quirúrgicas de reasignación de sexo y procesos de feminización o masculinización para la comunidad transexual, también es posible adelantar un trámite jurídico para lograr un cambio en el sexo a nivel de identificación civil. Este trámite no requiere adelantar una cirugía previa y, en ese sentido, puede ser utilizado por la comunidad transgénero para cambiar su identificación legal en la sociedad.

Esta posibilidad se abrió gracias a la Sentencia T-063 (2015) de la Corte Constitucional, en la cual se reconoció el derecho de las personas de solicitar la corrección del componente sexo en su registro civil. Con base en tal providencia, el Gobierno colombiano expidió el Decreto 1227 (2015), que reglamentó el procedimiento jurídico para la solicitud de dicha corrección, de modo que las personas se identificaran civilmente con el sexo que hubiesen escogido en ejercicio de su derecho fundamental al libre desarrollo de la personalidad, y sin que fuese necesario acudir a un largo proceso judicial o realizar algún tipo de intervención quirúrgica.

De esta forma, Colombia dio un paso adelante en el reconocimiento de los derechos de las personas transgénero, con lo cual se sumó a países como Argentina o Uruguay, donde se permite la modificación del sexo en los documentos de identidad sin que sean necesarios procedimientos quirúrgicos previos (Bockting y Keatley, 2011). Así, se cumplió con la declaración de reconocimiento de la Asociación Profesional Mundial para la Salud del Transgénero (WPATH por sus siglas en inglés), en la cual justamente se instó a los gobiernos del mundo a eliminar las barreras administrativas y los requisitos de cirugías previas para el reconocimiento de la identidad de las personas transgénero en sus documentos civiles (WPATH, 2015).

Esta reglamentación es una muestra de que el reconocimiento del transgenerismo en el país ha aumentado con el paso de los años, lo cual se ratifica con las cifras entregadas por la Registraduría Nacional del Estado Civil. En respuesta a la solicitud de información del número total de personas que han realizado el cambio del componente sexo desde el año 2015 (fecha de expedición de la normatividad que lo permitió) hasta el 22 de agosto de 2018, se conoció que iban en total 2.070 cambios del componente sexo. La mayoría de cambios han sido hacia el sexo femenino, tal como se describe en la tabla 1 :

Tabla 1. Cambio componente sexo en el registro civil

\begin{tabular}{ccc}
\hline Año & Sexo & Cantidad \\
\hline \multirow{2}{*}{2015} & Femenino & 288 \\
\cline { 2 - 3 } & Masculino & 176 \\
\hline
\end{tabular}




\begin{tabular}{ccc}
\hline \multirow{2}{*}{ Año } & Sexo & Cantidad \\
\hline \multirow{2}{*}{2016} & Femenino & 412 \\
\cline { 2 - 3 } & Masculino & 242 \\
\hline \multirow{2}{*}{2017} & Femenino & 370 \\
\cline { 2 - 3 } & Masculino & 200 \\
\hline \multirow{2}{*}{2018} & Femenino & 228 \\
\cline { 2 - 3 } & Masculino & 154 \\
\hline
\end{tabular}

Fuente: Registraduría Nacional del Estado Civil (2018).

Ahora bien, al crear la posibilidad de efectuar el cambio en la identificación civil de la población transgénero, se generan algunos impactos en el sistema de salud que busca atender y gestionar sus riesgos. Es importante la correcta identificación en la carga de enfermedades de las personas transgénero, pues al presentarse legalmente con un sexo diferente al de su nacimiento, las entidades del sistema no logran conocer la realidad biológica de la persona y por tanto no tienen en cuenta estrategias para reducir el impacto de patologías propias de cada sexo (cáncer de cérvix o de próstata entre otras). Adicionalmente, se presentan inquietudes sobre la forma de adelantar el registro clínico de las personas que cambian su sexo y nombre, así como la forma en que debe atenderse a la población sin generar discriminación durante el acto médico.

Otro punto complejo se produce en los valores que son girados por el Estado para que las aseguradoras del sistema financien los servicios de salud de las personas, pues estos varían dependiendo de la edad y el sexo.

El presente artículo inicia con un análisis sobre el concepto de transgenerismo para tener una definición clara a través de la cual se puedan establecer las consecuencias de este cambio en el sistema de salud en relación con la atención de los pacientes, la gestión de sus riesgos y los valores reconocidos por su aseguramiento. Se parte de la premisa de que están en juego los derechos fundamentales a la salud y al libre desarrollo de la personalidad de la población transgénero, y se finaliza con la percepción de algunos administradores en salud sobre este tema.

El presente análisis se realizó utilizando un enfoque metodológico de carácter cualitativo. A partir de la búsqueda de información se pudo indagar sobre el problema y plantear hipótesis. El tipo de investigación es, a su vez, documental, argumentativo y exploratorio, pues parte de conceptos desarrollados por la ciencia, la normatividad y la jurisprudencia. Se delimitó el objeto de estudio a los aspectos económicos y del sistema de salud de la comunidad LGTBI, especialmente transgénero, y a toda la literatura que promueve la identidad de género. Igualmente, se 
realizaron tres entrevistas a personas de cargos directivos en tres EPS del departamento de Antioquia. Luego de ser codificadas, estas entrevistas permitieron observar las percepciones de las administradoras sobre el tema para establecer las conclusiones de la investigación.

\section{IDENTIDAD DE GÉNERO, COMUNIDAD LGTBI Y PERSONAS TRANSGÉNERO}

La identidad de género es un concepto que se utiliza para referirse a la forma en que una persona se identifica con su sexo y las conductas desplegadas a raíz de este. Dentro de la identidad de género se encuentran la orientación sexual, la forma de vestir, el rol desempeñado en la sociedad, etc. Tiene como punto de partida histórico la heterosexualidad y el desempeño del rol femenino o masculino según la asignación de género que se realizó al nacer de acuerdo con las condiciones físicas de la persona. Sin embargo, en la actualidad abarca a todos los grupos de personas que no se encuentran conformes con el sexo asignado biológicamente, ni con el género que ese sexo les impone, ni con su orientación sexual, ni con los estándares de comportamiento que establece la sociedad (Lamas, 1996).

La Organización Mundial de la Salud (OMS) en su política sobre el género desarrolla algunos conceptos, entre ellos establece:

El término género se utiliza para describir las características de hombres y mujeres que están basadas en factores sociales, por su parte el sexo se refiere a las características que vienen determinadas biológicamente, por lo que las personas nacen con sexo femenino o masculino, pero aprenden a ser niñas y niños que se convierten en hombres y mujeres, es este comportamiento lo que compone la identidad de género y determina los papeles del mismo. (Organización Mundial de la Salud, 2002, p. 5)

Ahora bien, existe un grupo poblacional que ha venido cuestionando las identidades de género tradicionales: la comunidad LGTBI. Bajo este nombre se agrupan las personas lesbianas, gais, trans (transexuales y transgénero), bisexuales, intersexuales, entre otros.

Las manifestaciones de la identidad de género reconocidas actualmente pueden resumirse en la figura 1 .

En el grupo poblacional conocido como LGTBI también se encuentran los tran sexuales y los transgénero. Estos términos, aunque sean usados como iguales, en la teoría y en la práctica se refieren a personas en etapas distintas de su identidad sexual y del cambio que ello conlleva (Secretaría de Gobernación de México, 2016).

A diferencia de los conceptos explicados en la gráfica, el transgenerismo (para efectos de este documento "trans"), es la manifestación que hace referencia a las personas que cuando nacen — - y debido a su condición física — se les realiza cierta 


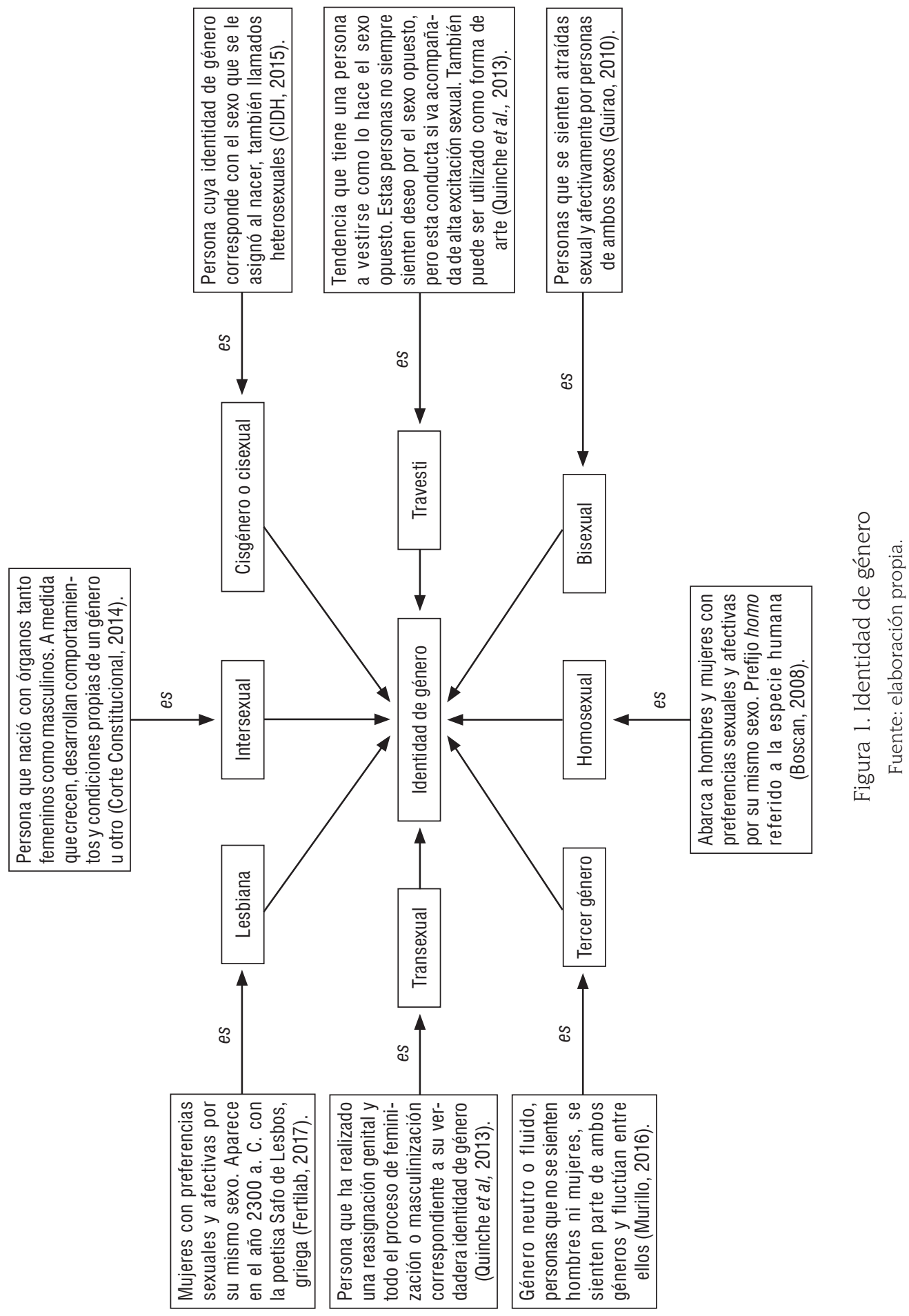


asignación de género, es decir, masculina o femenina. Sin embargo, a medida que se desarrollan como personas, se sienten identificadas con el género contrario. Empiezan a comportarse como lo haría una persona del sexo opuesto, al punto que en ocasiones cambian su nombre y el componente sexo en su documento de identidad a través de un trámite legal. También puede suceder que, al sentirse conformes con el cuerpo, no lleguen a la reasignación de sexo y no se realicen cambios corporales. Este tipo de personas, por lo general, no acude en busca de ayuda profesional para cambiar su aspecto biológico (Noseda, 2012).

No obstante, un problema que afronta la comunidad trans tiene que ver con la discordancia en los distintos significados que se dan a las palabras usadas para referirse a ellos. Por lo general, la literatura científica diferencia al transgénero y al transexual por el cambio realizado quirúrgicamente o no, respectivamente. Asimismo, en países como Argentina, la persona trans es llamada también travesti y con esta se refieren a cualquier individuo que haya empezado un tránsito, bien sea físico, hormonal, quirúrgico o solo legal. Es importante tener en cuenta que las personas trans se niegan a ser catalogadas con nombres dependiendo de su condición. Por ello, hay múltiples términos en distintos países del mundo para referirse a ellos y, asimismo, es variada la forma en que los expertos los abordan (Bockting y Keatley, 2011).

Siguiendo este hilo conductor, en Colombia el panorama no es muy distinto. En el ordenamiento jurídico se evidencia poco desarrollo legal para proteger a la población LGTBI, aunque realmente el desarrollo jurisprudencial de la Corte Constitucional ha abierto las discusiones de sus derechos respecto a diversos temas: la adopción de parejas del mismo sexo (Sentencia C-683, 2015), el matrimonio igualitario (Sentencia SU-214, 2016), el derecho a suceder de las parejas del mismo sexo (Sentencia T-717, 2011), entre otros.

Ahora bien, en el sistema de seguridad social existe una serie de barreras que impiden el acceso efectivo al goce del derecho fundamental a la salud. En este sentido, las personas trans enfrentan determinados obstáculos para el goce efectivo de su derecho en condiciones de igualdad. Se evidencian dos grandes problemas para el ejercicio de ese derecho en esta población, los cuales tienen que ver con las afectaciones a los estados de salud y las barreras en los servicios de salud.

\section{PRINCIPALES PROBLEMAS QUE AFRONTA LA POBLACIÓN TRANSGÉNERO EN MATERIA DE SALUD}

Los principales problemas que enfrenta la población trans se deben a factores individuales, familiares, sociales y del sistema de salud. Estas personas deben soportar que su identidad de género no se refleje en su identidad legal, y esto inicia un proceso de aceptación propia que puede derivar en problemas de aislamiento social, depresión, baja autoestima, desórdenes alimenticios, entre otros (Bockting y Keatley, 2011). 
Desde el mismo momento en que la persona trans inicia su proceso de aceptación propia empieza a requerir — además de sus capacidades individuales—, recursos familiares, sociales e institucionales para afrontar los problemas que se derivan de la disonancia entre su identidad de género y su identidad legal. Muchas personas trans que no son aceptadas por su comunidad terminan aislándose de sus hogares, en camino a la mendicidad, la prostitución o determinadas a ejercer actividades que se reconocen como propias de la población LGTBI, como el mercado de bienes y servicios cosméticos (Bockting y Keatley, 2011).

En general, las personas trans tienen que afrontar barreras relacionadas con el acceso a los servicios de educación, vivienda, salud y participación social. Las relacionadas con el acceso a los servicios de salud tienen que ver con la falta de educación al personal asistencial frente a la sensibilidad en el trato a personas que afrontan una transición de género, así como el acceso a servicios de alto costo o que no se encuentran cubiertos por los planes de beneficios (Barreda e Isnardi, 2004).

A lo anterior se suma la poca formación que obtiene el personal de salud sobre el tratamiento que debe darse a una persona trans, lo que se traduce en una débil incorporación del trato diferencial que debería darse desde el momento de la admisión a un servicio de salud. El médico, por ejemplo, debería tener la capacidad suficiente de entender a la persona trans como un ser en discordancia con su identidad de género, conocer todos y cada uno de los cambios a los que se ha sometido, qué órganos sexuales conserva, entre otros. Pero debido al desconocimiento, dentro de la atención médica se plantean preguntas, comentarios y expresiones inapropiadas, se genera demora, la atención termina derivándose de un médico a otro o finalmente no se presta. Todo esto genera que el paciente no quiera volver a solicitar los servicios (Mejía y Benavides, 2008).

Por otra parte, el coordinador del programa municipal de sida de Rosario, Argentina, manifiesta que en su experiencia personal ha podido evidenciar que las dificultades derivadas del proceso de adecuación corporal están relacionadas con prácticas que atentan contra la salud de las personas que se someten a este. Entre ellas se encuentra el uso indiscriminado de hormonas que incluye la ingesta de métodos anticonceptivos orales, entre dos y tres comprimidos al tiempo, o la inyección directa de hormonas, los cuales generalmente se prescriben de forma mensual o bimensual y son aplicados semanalmente. Finalmente se encuentra el suministro en diferentes partes del cuerpo de sustancias que ayudan al aumento del tamaño, como son aceites vegetales, de cocina o sustancias derivadas de la silicona no aptas para la aplicación en seres humanos (Presidencia de la Nación Argentina, 2015).

A modo de ejemplo, la ingesta desmedida de anticonceptivos usados principalmente para aumentar la producción de hormonas femeninas en un cuerpo masculino 
puede llevar al padecimiento de embolias, accidentes cardiovasculares, depresiones crónicas, migrañas, alteración de la función del hígado, diabetes mellitus, entre otros (Bockting y Keatley, 2011).

Respecto a las inyecciones de sustancias siliconadas o aceites, se evidencia que son aplicadas por personas que no están capacitadas para ello o que estas sustancias no son aptas para aplicación en humanos. Esto ha generado fuertes problemas de salud pública en varios países de Latinoamérica (Bockting y Keatley, 2011).

Otro de los riesgos que padecen las personas trans está catalogado como un problema de salud mental. Tiene que ver con los altos niveles de estrés, ansiedad y conductas suicidas a los que se ven expuestos, "como resultado del estigma asociado a la no conformidad de género, es decir, las divergencias de las normas y expectativas sociales tradicionalmente asociadas con el sexo asignado al nacer" (Mejía y Benavides, 2008).

Ahora bien, los determinantes sociales juegan un papel importante en los problemas a los que se ve enfrentada la comunidad trans, entre ellos el acoso, la discriminación y la exclusión de los ámbitos familiar, social, laboral, escolar, así como la creencia errónea de que son población altamente siniestrada en salud simplemente por haber iniciado su proceso de transición. Todo esto lleva a la población a ser víctima directa de violencia de género, padecer enfermedades de carácter mental, estar más expuestos a VIH y otras enfermedades de transmisión sexual, al consumo excesivo de alcohol o sustancias psicoactivas, así como problemas dentales, dermatológicos, del sueño, de nutrición, entre otros (Bockting y Keatley, 2011).

Finalmente, frente a la atención efectiva y de calidad en las diferentes instituciones o entidades, se tiene que

pocos médicos y otros profesionales de la salud han recibido, durante su formación, información sobre las necesidades de las personas trans. Por lo tanto, no es de extrañar que los profesionales experimenten incomodidad con lo desconocido cuando se encuentran con consultantes cuya identidad o expresión de género no corresponde con su sexo natal asignado. Además, los profesionales pueden haber adquirido información incorrecta, lo cual puede inhibir su capacidad para proporcionar una atención óptima a las personas trans. (Organización Panamericana de la Salud, 2011, p. 85)

Por otro lado, las mujeres trans son víctimas de distintos tipos de violencia al consultar los servicios de salud. La conducta violenta inicia con la discriminación por parte del personal, desde el vigilante de la entrada de la institución, quien en general no sabe cómo referirse a una mujer trans, y las personas de admisiones, quienes las llaman como hombres cuando se les llega a negar la atención o al momento de la misma las tratan de forma irrespetuosa (Lasso, 2014, p. 109). 
Puede afirmarse que el principal inconveniente frente a la atención radica en que

los médicos pueden no entender por qué las travestis se inyectan silicona, se involucran en el trabajo sexual o utilizan drogas. Los servicios de hospitalización son, a menudo, segregados por sexo, y las travestis son asignadas a la sala de los hombres, lo que plantea grandes desafíos. Esto lleva a muchas travestis a evitar totalmente la atención de la salud y, por lo tanto, no reciben atención preventiva o de intervención temprana. (Organización Panamericana de la Salud, 2011, p. 48)

Esto degenera entonces en que las personas trans, debido a la estigmatización y patologización que han sufrido, "experimentan miedo al rechazo, al tratamiento inadecuado o a la negación de servicios, por lo que prefieren, utilizar servicios privados o clandestinos, la automedicación o mantenerse fuera de los servicios de salud por completo" (Organización Panamericana de la Salud, 2011, p. 54).

A lo anterior se suma una estigmatización por el VIH o el sida, pues cuando consultan por razones distintas a esta patología, suelen ser inmediatamente relacionadas con el mismo, lo cual no solo les ofende, sino que les margina de la consulta y oculta "otros motivos de consulta como el cáncer, problemas de próstata, entre otros" (Lasso, 2014).

Teniendo en cuenta los conceptos acerca de identidad de género, especialmente el de transgénero, se plantearon los retos que se encuentran en la normatividad del sector de la salud ante la posibilidad de realizar una modificación en el documento de identidad sin someterse a una intervención quirúrgica, puntualmente en lo que se refiere a la gestión del riesgo en salud y al costo de su aseguramiento.

\section{CAMBIO EN LA IDENTIDAD CIVIL Y GESTIÓN DE RIESGO EN SALUD}

La posibilidad de cambio en el componente sexo de la identidad civil de las personas trans, lleva a plantearse algunas preguntas sobre sus efectos en los sistemas de información de las Instituciones Prestadoras de Servicios de Salud (IPS). ¿Si un transgénero cambia su sexo, nombre y número de identidad, debe reflejarse tal cambio en toda su historia clínica o solo se cambiará desde la modificación de los documentos en adelante? ¿Cómo se podrá conservar un histórico de atenciones con dos identidades diferentes, en caso de que solo se modifiquen los documentos después del cambio de sexo?

Frente a estas dudas, la Agencia Española de Protección de Datos (AEPD) ha indicado que, de acuerdo con la normatividad de tal país y siguiendo las necesidades lógicas del servicio de salud, toda la historia clínica debía registrarse a nombre de la nueva identidad de la persona trans, pero era necesario indicarse su sexo de nacimiento en cada uno de los episodios anteriores al cambio para que los profesionales tuviesen tal conocimiento (2011). Esta propuesta es bastante razonable, en la medida 
que permite una corrección conservando una nota en la historia para efectos de la atención de acuerdo con las condiciones biopsicosociales de cada persona trans.

No obstante, en el caso colombiano, la Resolución 1995 (1999), que regula el manejo de la historia clínica, establece en los artículos 5 y 18 que la misma no puede ser modificada o alterada una vez termina la atención. En este sentido, no se aplica totalmente lo manifestado por la AEPD, de modo que lo más recomendable es que los registros anteriores al cambio se conserven con la antigua identidad y los posteriores incorporen la nueva identidad. Ambos grupos de registros conforman una unidad a nivel informático para que pueda ser consultada de forma secuencial y sin perder el conocimiento histórico del paciente que permita brindarle una atención integral.

De igual forma, el cambio en la identidad no solo afecta el registro clínico, sino la forma como se adelanta la gestión de riesgo. Diversas normas han tratado la ges tión de riesgo en salud, desde el Plan Decenal de Salud Pública 2012-2021 hasta la Resolución 2626 (2019) sobre la Política Integral de Atención en Salud, en los cuales se ha apuntado que las EPS, las IPS y las entidades territoriales realicen intervenciones a nivel individual y colectivo para gestionar los riesgos a los que está expuesta la población, lo cual debe llevarse a cabo de forma integrada entre los actores, tal como se aprecia en la figura 2.

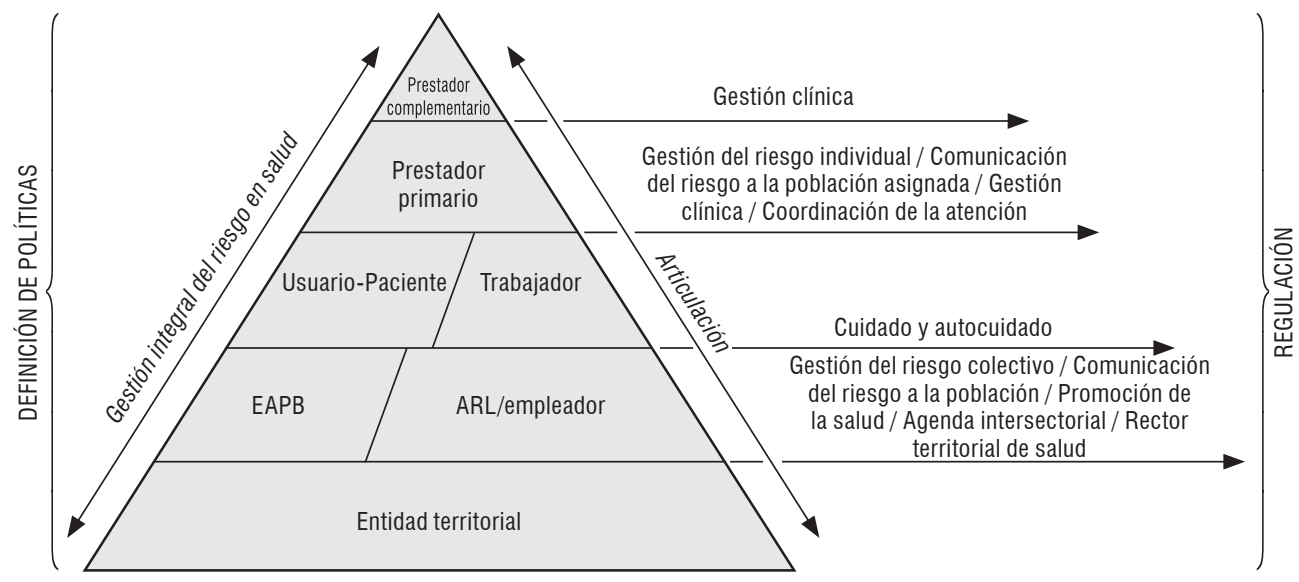

Figura 2. Gestión integral de riesgo en salud

Fuente: Ministerio de Salud y Protección Social (2018a, p. 18).

De acuerdo con lo planteado por el Ministerio de Salud, la Gestión Integral de Riesgo en Salud (GIRS) implica una serie de elementos y procesos, entre los cuales destacan la tamización, identificación y seguimiento de personas en riesgo de enfermedad, conformación de cohortes para el seguimiento de personas de acuerdo con los riesgos de enfermedad, análisis de riesgo de la población con enfoque diferencial, 
categorización y clasificación del riesgo de las personas, conformación de grupos de riesgo, intervención sobre la apropiación individual de riesgo y modificación del riesgo a través de acciones costo efectivas aplicadas por el personal de salud y prestadores de servicios (Ministerio de Salud y Protección Social, 2016).

Ahora bien, para la realización de estos procesos la variable sexo tiene relevancia, pues hace parte del enfoque diferencial con el cual se pretende materializar de forma más efectiva la intervención en cada persona, por lo cual el mismo Ministerio aclara que este enfoque "es una dimensión relativa, pues cada persona tiene su propio nivel y tipo de vulnerabilidad en función de situaciones socioeconómicas, culturales, políticas, religiosas, de género y personales" (Ministerio de Salud y Protección Social, 2016). De esta forma, para la aplicación de la GIRS, la identificación del grupo poblacional o del individuo, dependiendo de si se trata el componente colectivo o el individual, incluye factores que pueden variar sus condiciones, entre los cuales se encuentra el sexo.

Lo anterior tiene su fundamento en el hecho de que existe una serie de patologías propias del sexo femenino, como por ejemplo el cáncer de cérvix, y a su vez existen otras que ocurren solo con el sexo masculino, como el cáncer de próstata (Mohar et al., 1997). De esta forma, clasificar a una persona en sexo masculino o femenino tiene consecuencias sobre las medidas preventivas que se tomen para evitar el desarrollo de estas patologías. Si se piensa, por ejemplo, en la tamización de personas en riesgo de enfermedad, como etapa inicial de la GIRS, hacen parte de ella medidas como la citología anual en mujeres o el examen de antígeno prostático en los hombres a partir de los cincuenta años.

No obstante, en el caso de las personas transgénero que han cambiado la variable sexo en sus documentos de identidad, pero que conservan sus genitales de nacimiento, se presenta una desviación en el uso de la estrategia, pues los sistemas de información identificarán a las personas de acuerdo con sus documentos civiles, mas no con su realidad biológica. Esto lleva a que a un hombre trans con órganos femeninos no le sean evaluados los riesgos de cáncer de cérvix ni que a una mujer trans con órganos masculinos le tengan en cuenta el riesgo de cáncer de próstata. De esta forma, este grupo poblacional estaría invisibilizado para la estrategia de la GIRS en aquellas patologías sobre las que tenga incidencia su biología sexual, tanto en el componente colectivo como en el individual.

Esta situación provoca que los análisis predictivos de riesgo y las demás estrategias propias de la GIRS puedan presentar errores en su uso con las personas transgénero, y en ese sentido no sea fácil su aplicación, lo que llevaría a limitar el acceso del grupo poblacional a los servicios preventivos debido a la falta de adaptación de la estrategia a su realidad. Esta situación no encuentra un análisis en la normatividad 
vigente, ni en los documentos producidos hasta el momento por el Ministerio de Salud como director del sistema, pues en ellos no se aprecia la necesidad de contar con herramientas tecnológicas que permitan una mejor identificación de las personas trans para efectos de aplicar la estrategia. Existe incluso una ausencia de referencias a este grupo, lo cual indica que la problemática no ha sido evidenciada por el gobierno o los actores del sistema.

Adicionalmente, es necesario preguntarse si las patologías con carga prevalente en un sexo conservarían tal condición en el caso de una persona transgénero, toda vez que el uso de tratamientos hormonales podría terminar afectando dicha prevalencia. Esta situación requiere un análisis y estudio más profundo desde el punto de vista fisiológico y epidemiológico.

En este orden de ideas, un punto que debería tenerse en cuenta en la adaptación de la GIRS a las personas transgénero es la necesidad de contar con el dato del sexo biológico más allá del sexo con el cual se identifican legalmente, pues como se ha mencionado, el segundo puede variar sin que sea necesaria una intervención quirúrgica. Una alternativa es la inclusión de la variable sexo biológico en los análisis que deban adelantarse, tanto por los prestadores de salud, como por los aseguradores y los entes territoriales.

Adelantar la GIRS atendiendo al sexo biológico de una persona implicaría que, en el caso de las personas transgénero, no se dejen de lado las patologías que podrían amenazarlos, como las de cáncer de cérvix o de próstata ya mencionadas, pues con base en el conocimiento de la realidad fisiológica del individuo se podría llegar a una mayor efectividad de la estrategia. El problema que se ha venido describiendo radica en que no se puede tomar la variable sexo de la identificación como una verdad concordante con la biología del individuo, entonces la solución más sencilla será tomar la variable sexo biológico como categoría para abordar con un dato real al individuo y a la comunidad transgénero.

Una propuesta similar plantea Bockting y Keatley (2011) con el diseño de un formulario que podría ser utilizado para abordar a las personas trans en la admisión de la prestación de servicios. En tal formulario, la persona tendría la posibilidad de indicar cuál es su identidad de género actual, el sexo que le asignaron al nacer e incluso su nombre preferido. Lo anterior puede permitir una mayor confianza en la relación médico-paciente, como para generar un mejor conocimiento de la persona trans.

Tomando como ejemplo esta iniciativa, se puede plantear un uso similar en el modelo colombiano que permita adaptar la GIRS a la población transgénero. De esta manera, la implementación sería adecuada a las necesidades de esta población por parte no solo de los prestadores (como en la idea de Bockting y Keatley), sino también 
por las aseguradoras y los entes territoriales que no tienen un contacto directo con el usuario, pues solo conocen datos y cifras que lo representan.

Ahora bien, existe otra dificultad que se genera frente al cambio de identidad civil que pueden efectuar las personas transgénero, el cual apunta a la financiación de su aseguramiento por parte del sistema.

\section{CONSECUENCIAS DEL CAMBIO DE IDENTIDAD CIVIL RESPECTO A LA UPC}

De acuerdo con lo establecido en el literal f del artículo 156 de la Ley 100 (1993), las EPS reciben por parte del Estado una unidad de pago por capitación (UPC) por cada afiliado que tengan para financiar con ella el costo de los servicios que se requieran del plan de beneficios y el pago de incapacidades a que hubiese lugar.

La tarea de fijar el valor de la UPC le ha correspondido al Ministerio de Salud y Protección Social (en adelante MSPS), el cual anualmente la establece por vía reglamentaria, partiendo de que es un instrumento de política que se calcula con base en un estudio de suficiencia realizado a partir de la información de prestación de servicios reportada por las EPS. Gracias a esto es posible determinar el valor de la prima de riesgo que permita establecer condiciones de sostenibilidad económica de las entidades, y así garantizar la prestación del servicio contenido en el plan de beneficios del sistema (Ministerio de Salud y Protección Social, 2017).

Ahora bien, en los estudios de suficiencia publicados por el MSPS entre los años 2015 y 2018, dentro de sus variables de población a considerar han tenido en cuenta el sexo, y de ello ha resultado que, en la diferenciación de grupos por valor de UPC, no solo se clasifica de acuerdo con la edad, sino también se diferencia entre hombres y mujeres. Así, para el año 2020, con la Resolución 3513 (2019b), se fijó un valor más elevado (casi el doble) en el régimen contributivo para las mujeres entre los quince y los cuarenta y cuatro años, frente a los hombres en el mismo rango de edad, lo cual implica que las EPS reciban mayores recursos por ellas, tal como se muestra en la tabla 2.

Tabla 2. Estructura de UPC para régimen contributivo

\begin{tabular}{lcr}
\hline Grupo de edad & Estructura de costo & Valor año \\
\hline Menores de un año & 2,9679 & $2.649 .121,20$ \\
\hline $1-4$ años & 0,9530 & $850.640,40$ \\
\hline $5-14$ años & 0,3329 & $297.144,00$ \\
\hline $15-18$ años hombres & 0,3173 & $283.219,20$ \\
\hline $15-18$ años mujeres & 0,5014 & $447.544,80$ \\
\hline
\end{tabular}




\begin{tabular}{lcc}
\hline \multicolumn{1}{c}{ Grupo de edad } & Estructura de costo & Valor año \\
\hline 19-44 años hombres & 0,5646 & $503.956,80$ \\
\hline 19-44 años mujeres & 1,0475 & $934.988,40$ \\
\hline 45-49 años & 1,0361 & $924.814,80$ \\
\hline 50-54 años & 1,3215 & $1.179 .558,00$ \\
\hline 55-59 años & 1,6154 & $1.441 .893,60$ \\
\hline 60-64 años & 2,079 & $1.855 .695,60$ \\
\hline 65-69 años & 2,5861 & $2.308 .330,80$ \\
\hline 70-74 años & 3,1033 & $2.769 .976,80$ \\
\hline 75 años y mayores & 3,8997 & $3.480 .836,40$ \\
\hline
\end{tabular}

Fuente: Ministerio de Salud y Protección Social (2019b).

Lo anterior tiene su razón de ser en que a partir de los análisis se ha encontrado mayor consumo de servicios en mujeres. Así, en el estudio de suficiencia de 2015, se detectó que

(...) las mujeres presentan mayores porcentajes de uso de los servicios del sistema de salud que los hombres en la mayoría de los grupos de edad el mayor porcentaje de uso del servicio son los menores a 9 años de edad y las mujeres en edades de 20 a 40 años de edad. (Ministerio de Salud y Protección Social, 2015)

Igualmente, en el análisis de 2016 se determinó que los menores de cero a cuatro años y los mayores a de sesenta y cinco años presentaban mayores frecuencias de uso de los servicios de salud, teniendo en cuenta que los porcentajes de participación de las mujeres fueron mayores en todos los grupos de edad de quince y más años. Adicionalmente,

las acciones de promoción y prevención se han centrado en los menores de edad y las mujeres en edad fértil, con una menor extensión hacia la población adulta y adulta mayor, la cual desciende notoriamente a medida que avanza la edad. (Ministerio de Salud y Protección Social, 2016)

En el informe de 2017 se indicó que por cada cien mujeres había setenta y nueve hombres que accedían a los servicios de salud (Ministerio de Salud y Protección Social, 2017).

Finalmente, en el estudio de 2017 se concluyó que la estructura por sexo era similar a la observada en estudios pasados, setenta y ocho hombres por cada cien mujeres (Ministerio de Salud y Protección Social, 2017). 
Por otro lado, en el Análisis de Situación de Salud (ASIS), realizado por el Ministerio de Salud y Protección Social (2018b), se encontró que entre los años 2009 y 2017 se atendieron un total de 48.690 .085 personas, a quienes se prestaron 695.232.240 atenciones. La proporción de personas que recibieron atenciones es un 59 \% mayor en las mujeres que en los hombres (Ministerio de Salud y Protección Social, 2018b). Este mismo informe concluyó, frente a las patologías de alto costo, un comportamiento disímil entre hombres y mujeres. Por ejemplo, la enfermedad renal crónica en los estadios uno a cuatro afectaba en mayor porcentaje a las mujeres, mientras que en el estadio cinco era mayor en hombres. En la hipertensión arterial existe una prevalencia mayor en servicios de salud para las mujeres ( 0,070 por cada cien mil afiliadas) frente a los hombres (0,040 por cada cien mil afiliados). Con respecto a la diabetes mellitus hubo un comportamiento similar con una prevalencia mayor en servicios de salud para mujeres $(0,020$ por cada cien mil afiliados) frente a los hombres $(0,010$ por cada cien mil afiliados) (Ministerio de Salud y Protección Social, 2018b).

De esta forma, se ha concluido que las mujeres en cierto rango de edad resultan más costosas para el sistema, y por tal motivo en la Resolución 3513 (2019b) se fijó un valor de UPC mayor frente a los hombres para que de esa forma las EPS puedan garantizar su aseguramiento. Esta situación es comprensible y se encuentra sustentada en los estudios realizados por el MSPS. No obstante, esto lleva a un inconveniente cuando se trata de personas transgénero que han cambiado la variable sexo en su documento de identidad, toda vez que, como se ha manifestado, en Colombia no es necesario realizar una intervención quirúrgica para lograr un cambio en el documento de identidad, lo que implica que las personas pueden haber realizado la modificación legal y conservar su biología.

De acuerdo con la Resolución 4622 (2016), las EPS deben reportar a la Base de Datos Única de Afiliados (BDUA) los cambios en los documentos de identidad de sus afiliados, de tal modo que, ante una modificación en el registro del sexo, la EPS que conozca esta novedad deberá reportarla inmediatamente. Pero esto afecta también el valor que recibe la entidad por el afiliado. Si envía la novedad de cambio de mujer a hombre, obtendrá menos por concepto de UPC, aun cuando el afiliado conserva rasgos femeninos en su biología, puede que conserve sus órganos reproductores y puede incluso quedar en embarazo.

Pese a que la norma indica que se debe realizar el reporte, no manifiesta nada respecto a esta variación en la UPC. En los estudios de suficiencia y las normas que a ella se refieren no se encuentra que el MSPS se haya percatado de esta situación. Esto lleva a que los estudios presenten inconsistencias, pues al identificar en BDUA un hombre (su identificación legal), no están teniendo en cuenta que en realidad conserva una biología femenina, puede presentar un consumo de servicios mayor por anticonceptivos y eventualmente puede necesitar servicios de atención por embarazo. Estas situaciones no se están contemplando actualmente. 
Esta problemática no solo afecta los estudios de suficiencia, sino también los recursos que perciben las EPS para garantizar el aseguramiento de su población, pues con el cambio de UPC de acuerdo con el sexo legal terminarían recibiendo el monto por un hombre afiliado (menor al de la mujer), aunque tendrían que responder por el consumo de servicios propios del sexo femenino como los de la gestión y el parto, lo que dejaría una insuficiencia en esos casos.

Ahora bien, si para la realización de una correcta gestión de riesgo en salud se propone tomar el sexo biológico de la persona transgénero, igual regla debería aplicarse en cuanto al reconocimiento de la UPC, pues mientras conserve características femeninas, la EPS debería recibir el valor que corresponde a una mujer teniendo en cuenta que en promedio requieren más atenciones.

\section{PERCEPCIONES DE ALGUNOS ADMINISTRADORES DEL SECTOR SALUD RELACIONADAS CON EL FENÓMENO DEL TRANSGENERISMO}

Para tener una percepción general de lo que observan los administradores del sector salud en relación con la temática tratada, se consultó con personas que trabajan para tres de las principales EPS que operan en Antioquia (sus nombres no se indican, pues se garantizó su confidencialidad). Las tres personas indicaron que desde hace muchos años se ha interiorizado la diferencia entre género y sexo, y la importancia que tiene reconocer que el concepto de género debe incluirse en la atención en salud de las personas. En este sentido, el primer entrevistado expresó:

Definitivamente, yo creo que los que somos de mi edad tenemos más el concepto memoria de sexo, pero hay que aclarar que yo creo que, dentro de los sectores, el que más consciente y hace muchos años trabaja el tema de género es el sector salud. Para nosotros es muy claro la parte de género, para nosotros es muy claro. yo diría qué género se ha visibilizado más, precisamente, a raíz de la evolución que hemos tenido cultural en el país donde, digamos, primero, pues éramos hombre mujer, y, luego, empieza todas aquellas definiciones por esas partes pues conceptuales o hasta físicas, y de la famosa población, acuérdese cuando arranca LG y se le van poniendo las letricas. (CECR, comunicación personal, 12 de octubre de 2018)

Por su parte, el segundo entrevistado, en relación con la variante sexo y género para la gestión del riesgo en salud, manifestó:

Para gestionar el riesgo, que es en esencia biológico, claramente se tendría que tener prevalencia sobre el sexo por encima del género (...); el género en tema del contexto sociocultural es un derecho fundamental, o sea, es el libre desarrollo de la personalidad (...) Claramente, las personas tienen todo el derecho fundamental de desarrollar su género como bien le parezca, o sea, ese es un concepto sociocultural al cual se le tiene que dar plena validez. (GJAS, comunicación personal, 11 de octubre de 2018)

Finalmente, el tercer entrevistado frente al género y el sexo manifestó: "El género, obviamente, depende de muchas características, hoy en día no solo hay hombre 
y mujer, sino que hay géneros diferentes, hay muchas posibilidades, entonces son completamente diferentes" (JMA, comunicación personal, 17 de diciembre de 2018).

Todo esto permite concluir que en varias de las EPS en Antioquia existe conciencia frente a la diferencia entre género y sexo, y que es necesario realizar esta distinción en pro de poder garantizar derechos. A fin de cuentas, lo que se busca dentro del sistema de seguridad social en salud en el país es la garantía del derecho fundamental a la salud, independiente de las condiciones o características de cada persona.

Igualmente, cada uno de los entrevistados reconoció que la gestión del riesgo es un aspecto fundamental dentro de su operación diaria, en ello son concordantes sus respuestas. Esto se puede observar en la opinión del primer entrevistado, cuando afirma que "la importancia del riesgo, de gestionar el riesgo, es poder garantizar prevención en la enfermedad", pues, desde su punto de vista, "lo que ha buscado siempre todo el sector y la normativa les] que se pueda garantizar prevención" (CECR, comunicación personal, 12 de octubre de 2018). Se observa, además, en la idea del segundo entrevistado, según la cual "la gestión del riesgo es fundamental: si no hay ese enfoque, yo diría que no hay salud" (GJAS, comunicación personal, 11 de octubre de 2018). Finalmente, es posible evidenciarla en las palabras del entrevistado JMA, quien asegura que

Un riesgo es la enfermedad hoy, ya las causas se pueden gestionar, entonces es la base de la atención en salud. (...) La gestión de riesgo en salud va con la salud, porque es gestionar los riesgos que tienen las personas, es un tema que cada vez se habla más. (JMA, comunicación personal, 17 de diciembre de 2018)

Ahora bien, entre las personas entrevistadas, todas indicaron que en sus respectivas EPS se realiza un mapeo de la población, lo que lleva a generar cohortes que tienen exposición a riesgos comunes, es así como se puede identificar a las mujeres en edad fértil, hombres en edad para realizar tamizaje de próstata, personas con hipertensión, entre otros. De esta manera, la EPS realiza un constante seguimiento de los riesgos para finalmente impactar positivamente en la población.

Sin embargo, pese a que la población trans se ha ido visibilizando más con el paso de los años y muchos sectores, en especial el de la seguridad social, reconocen el derecho fundamental a la salud, han dejado de lado aspectos que no resultan muy problemáticos porque la población trans continúa siendo un grupo minoritario. Esta idea se evidencia en las siguientes expresiones de las personas entrevistadas:

Si no se puede identificar desde el documento identidad y desde la base de datos si una persona es hombre o mujer, claramente esto podría afectar a gran escala (...). Habría que identificar una manera de que, o no identificar, sino que, como ponerle también el deber a la persona que realice este cambio de sexo en su documento de identidad, que le manifieste a la entidad que lo hizo. (GJAS, comunicación personal, 11 de octubre de 2018). 
Acá no actualizan, acá no cumplen el deber siquiera de actualizar un dato, se van pal juzgado y allá actualizan cuanta vaina sea (...). Podés correr es el riesgo de no incluirlos en algo, y lo segundo pues realmente no hay como una..., un trato diferente de nada. (CECR, comunicación personal, 12 de octubre de 2018).

En este sentido, los entrevistados manifiestan que, si bien no existe una caracterización especial dentro de su gestión del riesgo para identificar a personas trans, por su sexo biológico pueden tener necesidades diferentes a las que implica su género legal. Están al tanto de los casos, bien sea que lleguen por orden de tutela o porque se reconozcan dentro de la entidad y, de esta manera, se reconocen los derechos que la persona requiere. Sin embargo, son enfáticos en manifestar que, a la fecha, la población es poca y no genera un problema significativo, pero a medida que la población vaya creciendo el deber de información se va haciendo más importante, especialmente de cara a los riesgos que se pueden generar por desconocer una situación particular.

Lo anterior implica que hay poca visualización de la situación, pues no se ha llevado a cabo una caracterización especial porque la población es poca. De igual forma, durante las entrevistas se preguntó si se conocían políticas o lineamientos del Gobierno colombiano para afrontar esta situación, y todos coincidieron en una respuesta negativa.

Ahora bien, en relación con la UPC, cada uno de los entrevistados expresó:

Podría afectar el consumo de servicios y, por ende, también el dinero que se tiene que invertir en ese consumo de servicios generando costos sobre una UPC de un hombre o viceversa (...). Sí se podría ver afectada la... el ingreso económico que podrían tener las aseguradoras en este tipo de casos. (CECR, comunicación personal, 12 de octubre de 2018)

Porque es que mire, si uno iría al detalle, ustedes saben que como está la UPC, cómo se construye con una nota técnica, unas frecuencias, uno diría, listo, pues por decir algo, imire, hombre que pasó a mujer de 25 años! En último, uno ve la UPC para esa edad, hombre - mujer, lógicamente es un poco más económica para el hombre, y un poco más para la mujer porque está en edad fértil, pero digamos que yo como EPS, póngale que tengamos 10 casos, pues realmente mantengo la política que mantenemos aquí, respetamos los derechos. (GJAS, comunicación personal, 11 de octubre de 2018)

Casos tan específicos, donde ya empiezan a hacerse intervenciones muy especializadas, ameritan que tenga una mirada y un cálculo diferencial, que uno dijera ojalá hubiera un cálculo diferencial para estos casos y también para otras cosas específicas que se salen de lo normal, de lo frecuente, deberían tener una UPC diferencial porque las intervenciones son muy altas, muy costosas y no son fáciles de realizar. (JMA, comunicación personal, 17 de diciembre de 2018). 


\section{CONCLUSIONES}

Pese a que el cambio de sexo en la identidad legal es un avance en el reconocimiento de los derechos de la población transgénero que pone a Colombia en la vanguardia de los países en este asunto, esta posibilidad no está en armonía con las reglas del sistema de salud y ello genera dificultades para la gestión del riesgo en salud de la población. En ese sentido, es necesario que los actores del sistema, principalmente el Ministerio de Salud y Protección Social en su rol de director y regulador, visibilicen esta situación y tomen medidas para acoger este cambio dentro del sistema.

Lo anterior puede llevarse a cabo a partir de una clasificación que atienda a la identificación legal y a la realidad biológica de las personas. De esta manera, se puede contar con datos fidedignos de la población que permitan conocer no solo su estado de salud, sino clasificarlos y elaborar estrategias de gestión de riesgo adecuadas a las patologías prevalentes por grupos etarios y sexo. Así, el análisis de la población no puede estar ligado solo a la identidad legal, ya que con ello se generaría una información poco certera en el grupo transgénero.

Frente a los registros clínicos, la norma colombiana limita la posibilidad de incorporar modificaciones a los mismos una vez finalizados, por lo que el cambio en la identidad legal no podría efectuarse retroactivamente. En este sentido, será necesario que las IPS cuenten con un software de historias clínicas que permita elaborar atenciones con la nueva identidad y enlazarlas con el histórico para no afectar la continuidad del registro del paciente transgénero.

De igual forma, al calcular el valor de la UPC que anualmente debe reconocerse a las EPS encargadas de garantizar el aseguramiento de la población, no debe tomarse el consumo de servicios desde la variable de identidad legal, ya que se presentaría la misma imprecisión. Esto se suma a que, al contar en el país con una UPC diferencial por sexo y grupo de edad, el dato para tener en cuenta debe ser el sexo biológico, pues es el factor que lleva a generar un mayor consumo de servicios, por lo que el cambio en el sexo legal no debería afectar el pago de UPC.

Estas situaciones no parecen estar identificadas por las EPS, lo cual se aprecia en las entrevistas, pues en ninguna de ellas se encontraron estrategias para afrontar estos puntos, y los entrevistados indicaron que no conocen políticas del Gobierno para resolver esta situación.

\section{REFERENCIAS}

Agencia Española de Protección de Datos. (2011). Informe 0268. https://www.aepd.es/informes/ historicos/2011 0268.pdf 
Asociación Mundial para la Salud del Transgénero, WPATH. (2015). Declaración WPATH 2015 sobre el reconocimiento de identidad. https://www.wpath.org/policies.

Barreda, V. e Isnardi, V. (2004). Travestismo y prevención del VIH/sida: reacomodando algunos conceptos [ponencia]. VI Jornadas de Sociología, Buenos Aires, Argentina. http://cdsa.aacademica.org/000 045/223.pdf.

Bockting, W. y Keatley, J. (2011). Por la salud de las personas trans. Elementos para el desarrollo de la atención integral de personas trans y sus comunidades en Latinoamérica y el Caribe. Organización Panamericana de la Salud. https://www.paho.org/arg/images/gallery/Blueprint\%20Trans\%20Espa\%C3\%83\%C2\%Blol. pdf?ua $=1$.

Boscan, E. (2008). Homosexualidad: los errores de concepto. Revista trabajo social UNAM, (18), 56-61.

Comisión Interamericana de Derechos Humanos. (2015). Violencia contra personas LGTBI. Organización de Estados Americanos. http://www.oas.org/es/cidh/informes/pdfs/ViolenciaPersonasLGBTI.pdf

Corte Constitucional de Colombia. (2011, 22 de septiembre). Sentencia T-717 (Luis Ernesto Vargas Silva, M. P.). http://www.corteconstitucional.gov.co/relatoria/2011/t-717-11.htm

Corte Constitucional de Colombia. (2014, 28 de agosto). Sentencia T-622 (Jorge Ignacio Pretelt Chaljub, M.P.) https://www.corteconstitucional.gov.co/relatoria/2014/t-622-14.htm

Corte Constitucional de Colombia. (2015, 4 de noviembre). Sentencia C-683 (Jorge Iván Palacio Palacio, M. P.). http://www.corteconstitucional.gov.co/RELATORIA/2015/C-683-15.htm

Corte Constitucional de Colombia. (2016, 28 de abril). Sentencia SU-214 (Alberto Rojas Ríos, M. P.). http://www.corteconstitucional.gov.co/relatoria/2016/su214-16.htm

Corte Constitucional de Colombia. (2015, 2 de febrero). Sentencia T-063 MARÍA (Victoria Calle Correa, M. P.). http://www.corteconstitucional.gov.co/RELATORIA/2015/T-063-15.htm

Guirao, M., (2010). Informe Kinsey. http://psicoterapeutas.eu/informe-kinsey/

Fertilab. (2012). Historia de la homosexualidad. Consultado el 9 de noviembre de 2018. http://www.fertilab. net/ginecopedia/sexualidad/homosexualidad/historia_de_la_homosexualidad_1.

Lamas, M. (2000). Diferencias de sexo, género y diferencia sexual. Cuicuilco, 7(18), 95-118 https://revistas. inah.gob.mx/index.php/cuicuilco/article/view/360

Lasso, R. A. (2014). Transexualidad y servicios de salud utilizados para transitar por sexos-géneros. Revista CES Psicología, 7(2), 108-125.

Mejía, A. y Benavides, L. (2008). Barreras de acceso a servicios de salud de las personas lesbianas, gay, bisexuales y transgénero en Bogotá D.C. http://www.sdp.gov.co/sites/default/files/barreras_acceso_servicios_ salud_personas_lgbt_2008.pdf

Ministerio de Salud y Protección Social. (2019). Resolución 2626. Por la cual se modifica la Política de Atención Integral en Salud - PAIS y se adopta el Modelo de Acción Integral Territorial - MAITE. https://www.minsalud. gov.co/Normatividad_Nuevo/Resoluci\%C3\%B3n\%20No.\%202626\%20de\%202019.pdf

Ministerio de Salud y Protección Social. (2019b). Resolución 3513. Por la cual se fijan los recursos de la Unidad de Pago por Capitación - UPC para financiar los servicios y tecnologías de salud, de los Regímenes Contributivo y Subsidiado para la vigencia 2020 y se dictan otras disposiciones. https://www.minsalud.gov. co/Normatividad_Nuevo/Resolucion\%20No.\%203513\%20de\%202019.pdf 
Ministerio de Salud y Protección Social. (2018a). Gestión Integral del Riesgo en Salud: Perspectiva desde el Aseguramiento en el contexto de la Política del Modelo Integral de Atención en Salud. https://www.minsalud. gov.co/sites/rid/Lists/BibliotecaDigital/RIDE/VP/DOA/girs-prespectiva-desde-aseguramiento.pdf

Ministerio de Salud y Protección Social. (2018b). Análisis de Situación de Salud (ASIS) Colombia, 2017. https:// www.minsalud.gov.co/sites/rid/Lists/BibliotecaDigital/RIDE/VS/ED/PSP/asis-nacional-2017.pdf

Ministerio de Salud y Protección Social. (2017). Estudio de sostenibilidad del aseguramiento en salud para el año 2017. https://www.minsalud.gov.co/sites/rid/Lists/BibliotecaDigital/RIDE/VP/FS/estudio-desostenibilidad-2017.pdf

Ministerio de Salud y Protección Social. (2016). Estudio de suficiencia y de los mecanismos de ajuste de riesgo para el cálculo de la unidad de pago de capitación para garantizar el Plan de Beneficios en Salud para el año 2016. https://www.minsalud.gov.co/sites/rid/Lists/BibliotecaDigital/RIDE/VP/RBC/estudiosuficiencia-upc-2016.pdf

Ministerio de Salud y Protección Social. (2016). Resolución 4622. Por la cual se establece el reporte de los datos de afiliación al Sistema General de Seguridad Social en Salud, a planes voluntarios, Regímenes Especial $y$ de Excepción y de las personas privadas de la libertad bajo la custodia y vigilancia del Inpec. https://www. minsalud.gov.co/sites/rid/Lists/BibliotecaDigital/RIDE/DE/DIJ/resolucion-4622-de-2016.pdf

Ministerio de Salud y Protección Social. (2015). Estudio de suficiencia y de los mecanismos de ajuste de riesgo para el cálculo de la unidad de pago de capitación para garantizar el Plan Obligatorio de Salud para el año 2015..https://www.minsalud.gov.co/sites/rid/Lists/BibliotecaDigital/RIDE/VP/RBC/estudiosuficiencia-2015.pdf

Ministerio de Salud. (1999). Resolución 1995. Por la cual se establecen normas para el manejo de la Historia Clínica. https://www.minsalud.gov.co/Normatividad_Nuevo/RESOLUCI\%C3\%93N\%201995\%20 DE\%201999.pdf

Mohar A, Frías-Mendivil, M., Suchil-Bernal, L., Mora-Macias, T., G. de la Garza, J. (1997). Epidemiología descriptiva de cáncer en el Instituto Nacional de Cancerología de México. Salud Pública de México 39(4), 253-258.

Murillo, S. (2016, 21 de junio). ¿Qué es el género neutro o tercer género? Ella hoy.http://www.ellahoy.es/ pareja/articulo/que-es-el-genero-neutro-o-tercer-sexo/247277/

Noseda Gutierrez, J. (2012). Muchas formas de transexualidad: diferencias de ser mujer transexual y de ser mujer transgénero. Revista de Psicología, 21(2), 7-30.

Organización Mundial de la Salud. (2002). Integración de las perspectivas de género en la labor de la OMS. Política de la OMS en materia de género. https://www.who.int/gender/mainstreaming/ESPwhole.pdf

Organización Panamericana de la Salud. (2011). Por la salud de las personas trans. Elementos para el desarrollo de la atención integral de personas trans y sus comunidades en América Latina y el Caribe. https://www.paho. org/arg/images/gallery/Blueprint\%20Trans\%20Espa\%C3\%83\%C2\%Blol.pdf.

Presidencia de la Nación Argentina. (2015). Atención de la salud inicial de personas trans. Guía para equipos de salud. http://www.msal.gob.ar/images/stories/bes/graficos/0000000696cnt-guia-equipos-atencionSalud\%20integral-personas-trans.pdf.

Presidencia de la República de Colombia. (2015, 4 de junio). Decreto 1227 de 2015, por el cual se adiciona una sección al Decreto número 1069 de 2015, Único Reglamentario del Sector Justicia y del Derecho, relacionada 
con el trámite para corregir el componente sexo en el Registro del Estado Civil. Diario oficial n. ${ }^{\circ}$ 49532. http:// www.suin-juriscol.gov.co/viewDocument.asp?ruta=Decretos/30019850

Quinche, M.F. y Peña, R.P. (2013). El derecho judicial de la población LGTBI y de la familia diversa. Legis y Universidad del Rosario.

Secretaría de Gobernación de México. (2016). ¿Quées la identidad de género? https://www.gob.mx/segob/ articulos/que-es-la-identidad-de-genero?idiom=es

Registraduría Nacional del Estado Civil. (2018). Respuesta solicitud de información estadística con radicado Formulario de Atención al Ciudadano: 21471392.

República de Colombia. (1993). Ley 100. Por la cual se crea el sistema de seguridad social integral y se dictan otras disposiciones. http://www.secretariasenado.gov.co/senado/basedoc/ley_0100_1993.html 